\title{
ELONGATED DISKS AND THE SCATTER IN THE TULLY-FISHER RELATION
}

\author{
MARIJN FranX \\ Harvard-Smithsonian Center for Astrophysics, 60 Garden Street, Cambridge, MA 02318 \\ AND \\ TIM DE ZEEUW \\ Sterrewacht, Huygens Laboratorium, Postbus 9513, 2300 RA Leiden, The Netherlands \\ Received 1991 December 24; accepted 1992 April 9
}

\begin{abstract}
It is often speculated that the dark halos of spiral galaxies are triaxial or prolate, with the long axis pointing in the plane of the disk. This would produce a potential that is elongated in the plane of the disk. We explore the signatures produced by such deviations from axisymmetry. We find observable effects in the photometry and kinematics of the stars and gas in the disk, if substructure like bars and spiral arms can be ignored. These effects include ellipticity gradients and position angle twists in disks, velocity gradients along the apparent minor axis of the disk, differences between kinematically determined inclinations and photometrically determined inclinations, and systematic residuals in circular orbit fits to the velocity field.

Noncircular gas motions produce significant scatter in the Tully-Fisher relation, amounting to $0.46 \mathrm{mag}$ if the ellipticity of the potential in the plane of the disk is 0.10 . Thus, small deviations from axisymmetry are sufficient to produce all or most of the observed scatter in the Tully-Fisher relation. The ellipticity of the potential in the plane of the disk must be less than 0.10 and most likely lies in the range $0-0.06$. This range corresponds to an ellipticity of the density distribution of the halo between 0 and 0.16 , if halos are the dominant mass component. The constraint on the shape of the dark halo is less tight in "maximum disk" models for spiral galaxies, since in these models the disk material significantly circularizes the total potential.

These results imply that use of detailed kinematic information may reduce the observed scatter in the TullyFisher relation by $0.1 \mathrm{mag}$ or more.
\end{abstract}

Subject headings: galaxies: distances and redshifts - galaxies: kinematics and dynamics galaxies: photometry

\section{INTRODUCTION}

Numerical simulations of galaxy formation produce halos with strongly triaxial-prolate shapes (Frenk et al. 1988; White \& Ostriker 1990; Dubinski \& Carlberg 1991). Spiral disks embedded in such halos are elliptical. There are indications from photometry that disks are nonaxisymmetric (e.g., Binney \& de Vaucouleurs 1981; Grosbøl 1985; Bertola, Vietri, \& Zeilinger 1991). Kuijken \& Tremaine (1991) review the recent work on this issue and conclude that the ellipticity of the disks may be of the order of 0.10 . A problem with these photometric analyses is that they may be influenced by spiral arms and bars. In this Letter we explore the observable effects of elongated disks on the kinematical properties of spirals and derive an upper limit on the ellipticity.

We assume that the total potential of the galaxy has an ellipticity independent of radius, and has a flat rotation curve. We use first-order epicyclic theory to calculate the observable properties of a disk in this potential $(\S 2)$ and then investigate the statistical effects on the Tully-Fisher relation. The observed low scatter in this relation is used to put a tight constraint on the overall ellipticity of the potential $(\$ 3)$. The result is discussed in $\S 4$.

\section{OBSERVABLE CONSEQUENCES OF ELONGATED DISKS}

We assume that both the gaseous and stellar components of disks are supported by rotation. Thus any pressure terms are ignored, and all material moves on closed orbits in the combined potential of disk and halo. These orbits are uniquely defined by the shape of the potential and the figure rotation. The same is true for the resulting velocity field.

First-order epicyclic theory gives a good approximation to the closed orbits when the elongation of the potential is small (Gerhard \& Vietri 1986; Binney \& Tremaine 1987). For a potential with a flat rotation curve, i.e., $V=\frac{1}{2} v_{c}^{2} \log m^{2}$, where $m^{2}=x^{2}+y^{2} / q^{2}$, and $q$ is a constant, the simple closed orbits are approximately elliptic, with ellipticity $\epsilon_{R}=1-r_{\min } / r_{\text {maj }}=$ $\epsilon$. Here $\epsilon \equiv 1-q$ is the ellipticity of the potential, and $r_{\max }$ and $r_{\min }$ are the major and the minor axis of the orbit, which is elongated perpendicular to the elongation of the potential. The velocity contrast along the orbit is given by $\epsilon_{v}=$ $1-v_{\min } / v_{\text {maj }}=2 \epsilon$. The maximum velocity $v_{\max }$ is reached at $r_{\min }$.

The observable properties of gas on closed orbits in a variety of triaxial potentials are discussed by Lees $\&$ de Zeeuw (1992) and Franx, van Gorkom, \& de Zeeuw (1992), using the epicyclic approximation. Detailed derivations of the expressions given below can be found in these papers.

\subsection{Photometric Parameters}

The surface density of the disk is elongated in the same direction as the orbits. Because the orbital velocity is not constant, the surface density also varies along each orbit. The resulting ellipticity $\epsilon_{D}$ of a disk with an exponential profile $\Sigma(R) \propto \exp \left(-R / R_{D}\right)$ embedded in the potential $V$ with $\epsilon$ constant, is, to first order,

$$
\epsilon_{D}(R)=\left(1+R_{D} / R\right) \epsilon .
$$




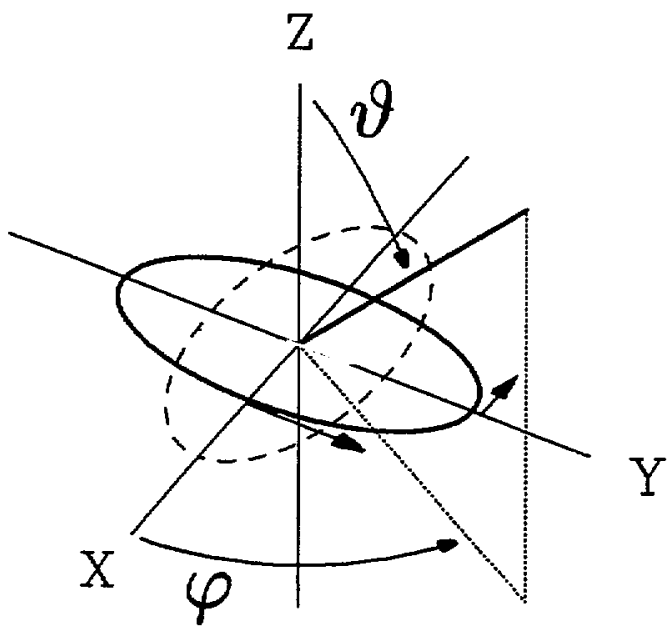

FIG. 1.-The viewing angles $\vartheta$ and $\varphi$. The orientation of the potential is indicated by the dotted ellipse. A closed orbit for the gas and stars is oriented perpendicular to the potential, as shown by the solid ellipse. The velocities along the elliptic orbits vary and are largest at the intersection with the $X$-axis and smallest at the $Y$-axis (as indicated by the arrows).

This equation holds only for $\epsilon_{D}(R) \ll 1$ and shows that the disk is more elongated than the orbits, but that at large distances the ellipticity of the disk approaches that of the orbits.

When the disk is observed from a direction specified by the spherical polar coordinates $(\vartheta, \varphi)$ (see Fig. 1), it has an ellipticity gradient, and a position angle twist: the position angle of the apparent major axis varies with radius. Expressions for the apparent ellipticity $\tilde{\epsilon}_{D}$ of the disk and for the position angle PA of the minor axis of the disk with respect to the projected normal to the plane are given in Table 1 .

When the disk is deprojected under the standard assumption of circular symmetry, the inferred parameters differ from the intrinsic parameters. Specifically, the estimated inclination $\hat{\vartheta}$ differs from the real inclination $\vartheta$. The deprojected surface density $\widehat{\Sigma}$ deviates similarly from the true surface density $\Sigma$, as does the derived scale length $\hat{R}_{D}$. The differences between these parameters depend on the viewing angles, and on the value of $\epsilon$, and expressions for them are given in Table 1 .

\subsection{Kinematic Effects}

The noncircular motion causes distinct signatures in the observed velocity field of the disk. There is a velocity gradient along the apparent minor axis of the disk for nearly all viewing angles. Table 1 lists the rotation velocity $v_{\text {minor }}$, which is half the velocity amplitude along the photometric minor axis. Fur-

TABLE 1

Observables of Elongated Disks ${ }^{a}$

\begin{tabular}{|c|c|c|}
\hline Observable & $\sin 2 \varphi$ & $\cos 2 \varphi$ \\
\hline 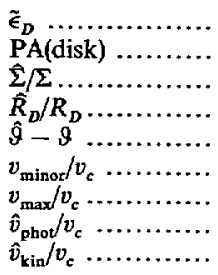 & $\begin{array}{c}\ldots \\
-\epsilon_{D} \sin 2 /(\sin \vartheta \tan \vartheta) \\
\cdots \\
\cdots \\
\cdots \\
\left(\epsilon+\epsilon_{D} / \tan ^{2} \vartheta\right) \sin \vartheta \sin 2 \\
\ldots \\
\cdots \\
\cdots\end{array}$ & $\begin{array}{c}1-\cos \vartheta\left(1-\epsilon_{D} \cos 2 \varphi\right) \\
\ldots \\
1-\epsilon_{D} \cos 2 \varphi \\
1+\frac{1}{2} \epsilon_{D} \cos 2 \varphi \\
\epsilon_{D} \cos 2 \varphi / \tan \vartheta \\
\ldots \\
\sin \vartheta(1-\epsilon \cos 2 \varphi) \\
1-\left(\epsilon+\epsilon_{D} / \tan ^{2} \vartheta\right) \cos 2 \\
1-\epsilon \cos 2 \varphi\end{array}$ \\
\hline
\end{tabular}

These expressions are valid for large inclinations $\vartheta$ and small $\epsilon$. thermore, the maximum velocity observed, $v_{\max }$, depends not only on inclination, but also on the orientation of the galaxy with respect to the line of sight. When we look down the long axis of the gas orbits, we measure a higher velocity than when we look down the short axis.

The integrated line profile of the entire disk also differs from that of a circular disk. The most significant effect is the change in the total width $W$, which is now given by $W=2 v_{c}(1-$ $\epsilon \cos 2 \varphi) \sin \vartheta$. The shape of the normalized line profile deviates slightly from that of a circular disk with the same total width, but the effect is only of order $\epsilon$.

The intrinsic kinematics of spiral disks are generally derived from the observed velocity field assuming circular symmetry. Two methods for determining the inclination of the disk are popular. In the first one, the photometry is used to estimate the inclination, and then the intrinsic circular velocity $\hat{v}_{\text {phot }}$ is estimated from the integrated line width, or from the velocity profile along the major axis. This method introduces two errors if the velocity field is noncircular: the photometric inclination differs from the real inclination, and the velocity amplitude deviates from $v_{c} \sin \vartheta$. Both effects correlate with $\cos 2 \varphi$, i.e., the orientation of the long axis of the potential in the plane of the disk with respect to the line of sight, and they tend to amplify each other. In the second method, the two-dimensional velocity field is modeled, usually with a velocity field based on motion in circular rings of varying inclination. The inclination and position angle of each ring are derived from the model fit. If the disk is flat, but elliptic, then this fitting procedure gives an inclination which is equal to the true inclination to first order (Franx et al. 1992). The deprojected circular velocity $\hat{v}_{\text {kin }}$ therefore is a better estimate of the intrinsic circular velocity than $\hat{v}_{\text {phot }}$.

Table 1 shows that all of the photometric and kinematic effects correlate with either $\epsilon \cos 2 \varphi$ or $\epsilon \sin 2 \varphi$. The dependence on inclination $\vartheta$ is included in the table, but the formulae are correct only for large $\vartheta$, i.e., for nearly edge-on systems. The $\epsilon \cos 2 \varphi$ term measures the difference in elongation in directions along and perpendicular to the line-of-sight, whereas the $\epsilon \sin 2 \varphi$ term measures the difference in elongation in the two directions at $45^{\circ}$ to the line of sight. We note that most of the observable effects of elongated disks correlate with $\epsilon \cos 2 \varphi$. The minor axis rotation $v_{\text {minor }} / v_{c}$ is probably the easiest to measure, but it gives information on $\epsilon \sin 2 \varphi$ only.

\section{OBSERVATIONAL CONSTRAINTS ON THE ELLIPTICITY OF THE POTENTIAL}

The best constraint on the shape of the potential in the disk of spiral galaxies comes from the scatter in the relation between luminosity and circular velocity (Tully \& Fisher 1977). An elongated potential introduces scatter in the Tully-Fisher relation for two reasons. First, the observed width of the line profile depends on the orientation of the long axis of the potential with respect to the line of sight (Table 1). Second, the photometric inclination differs from the real inclination. The latter effect is not relevant if the kinematic inclination is used to deproject the line profile. Both effects depend on $\cos 2 \varphi$ and amplify each other. As a result, the derived circular velocity $\hat{v}_{c}$ differs from the intrinsic circular velocity $v_{c}$, and depends not only on the intrinsic parameter $v_{c}$, but also on the accidental viewing angles $\vartheta$ and $\varphi$. We have computed the dispersion in the quantities $\hat{v}_{\text {kin }} / v_{c}$ and $\hat{v}_{\text {phot }} / v_{c}$ (see Table 1 ) by integrating over all viewing angles. Most authors exclude galaxies with small inclination $\vartheta$ (nearly face-on), because the inclination 


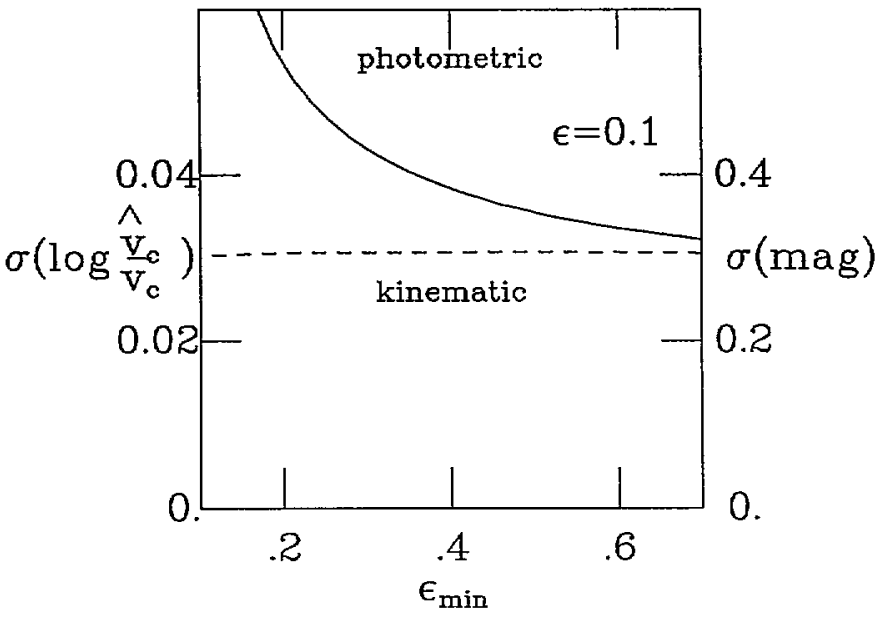

Fig. 2.-The expected scatter in the observed circular velocity $\hat{v}_{c}$ caused by an elongated potential as a function of lower limit in apparent ellipticity $\tilde{\epsilon}_{\text {min }}$. The solid line shows the scatter for circular velocities based on photometric inclinations, the dashed line for circular velocities based on kinematic inclinations. The intrinsic ellipticity of the potential is taken to be 0.10 . The righthand axis indicates the resulting scatter in the Tully-Fisher relation in magnitudes, assuming a relation of the form $L \propto v^{4}$.

corection becomes very large. In practice a lower limit is imposed on the apparent ellipticity of the disk $\tilde{\epsilon}_{D}$, and this then excludes certain viewing angles. In Figure 2 we illustrate how the scatter depends on the lower ellipticity limit $\tilde{\epsilon}_{\min }$, for a potential with ellipticity $\epsilon=0.10$. The scatter in the circular velocities based on kinematic inclinations is always lower than the scatter in the circular velocities derived from photometric inclinations. The scatter increases linearly with $\epsilon$ for $\epsilon \ll \tilde{\epsilon}_{\text {min }}$.

The scatter in the derived circular velocities produces scatter in the Tully-Fisher relation. For a relation of the form $L \propto v^{4}$, the scatter in the magnitude is $\sigma(\mathrm{mag})=10 \sigma\left[{ }^{10} \log \left(\hat{v} / v_{c}\right)\right]$. This is indicated on the right vertical axis of Figure 2 . An ellipticity of 0.10 produces a scatter in ${ }^{10} \log \left(\hat{v}_{\text {phot }} / v_{c}\right)$ of 0.046 for an ellipticity cutoff $\tilde{\epsilon}_{\min }=0.29$, and a corresponding scatter in the Tully-Fisher relation of $0.46 \mathrm{mag}$. This large value is caused by the steep slope of the relation.

The values quoted in the literature for the observed scatter in the Tully-Fisher relation differ by more than a factor of two: between 0.2 mag (Bothun \& Mould 1987) and 0.7 mag (KraanKorteweg et al. 1988). Part of this variation is due to differences in data quality, bandpasses, and data reduction pro- cedures. More recent studies give an observed scatter between 0.25 and $0.48 \mathrm{mag}$ in red bandpasses. Table 2 summarizes the various quoted values and also lists the ellipticity cutoffs that were used. We have calculated for each case the value of the disk ellipticity $\epsilon_{D}$ that could cause all the observed scatter. It is astonishing that most inferred values lie between 0.08 and 0.10 , despite the large range in $\sigma(\mathrm{mag})$. Apparently, the differences between the scatter reported in a variety of studies can be explained by an ellipticity of the potential of 0.09 , and differences in method, selection criteria, and slopes. This by itself might be taken as evidence that the scatter is largely produced by the ellipticity of the potential. We return to this issue below.

Detailed velocity maps of spirals can be used to detect noncircular potentials in individual galaxies. Many spiral galaxies show kinematic position angle twists, which can be due to either changes in ellipticity of the orbits or to warps. It should be possible to distinguish between the two cases by detailed model fitting, but this procedure has not been applied yet to large data samples. Bosma (1981) found kinematic twists in his sample, but he argued that many of the twists were caused by warps. Only seven of his 35 galaxies were interpreted to be oval; the twisting for these galaxies was typically $10^{\circ}-20^{\circ}$. The inferred mean twisting of $2^{\circ}-4^{\circ}$ can be explained by an ellipticity of 0.03 , if the inner parts are axisymmetric. Further constraints can be derived from the differences in inclinations and position angles from photometry and kinematic observations. We have seen in the above that such differences are expected for galaxies with triaxial potentials. Begeman $(1987,1989)$ has been the only author to apply a fully automated fit to the kinematic data. He fitted circular streaming models to eight galaxies. His results imply a mean inclination difference of $4^{\circ}$ and a mean position angle difference of $3^{\circ}$. The errors in the data are of comparable size. The predictions for a potential with an ellipticity of 0.10 are $3^{\circ}$ and $5^{\circ}$, respectively, roughly in agreement with the observed values. The small size of the sample and the uncertain observational errors make it difficult to provide strong constraints.

Finally, surface photometry can be used to derive the intrinsic ellipticity of disks. The presence of bulges, bars, spiral arms, and dust makes it nearly impossible to identify the ellipticity gradients and position angle twists in individual spiral disks caused by triaxial halo potentials. However, an overall elongation of the disk can be detected in the distribution of the apparent axial ratios in a complete sample of spiral galaxies.

We have analyzed the ellipticity distribution of nearly 600

TABLE 2

Scatter in the Tully-Fisher Relation and the Maximum Allowed Ellipticity of the Potential $\epsilon_{\max }$

\begin{tabular}{|c|c|c|c|c|c|c|c|}
\hline $\begin{array}{l}\text { Authors } \\
\text { (1) }\end{array}$ & $\begin{array}{c}\text { Sample } \\
(2)\end{array}$ & $\begin{array}{l}\text { Color }^{a} \\
\text { (3) }\end{array}$ & $\begin{array}{l}\text { Method }^{b} \\
\text { (4) }\end{array}$ & $\begin{array}{c}\tilde{\epsilon}_{\min }^{c} \\
(5)\end{array}$ & $\begin{array}{c}\text { Slope }^{d} \\
(6)\end{array}$ & $\begin{array}{c}\sigma(\mathrm{m})^{\mathrm{e}} \\
(7)\end{array}$ & $\epsilon_{\max }^{f}$ \\
\hline Aaronson et al. 1986 . & Clusters & $\mathbf{H}$ & Phot & 0.29 & 10 & 0.45 & 0.10 \\
\hline \multirow[t]{2}{*}{ Pierce \& Tully $1988 \ldots \ldots \ldots \ldots$} & Virgo & R, I & Phot & 0.13 & 8 & 0.48 & 0.08 \\
\hline & Ursa Major & R, I & Phot & 0.13 & 8 & 0.30 & 0.06 \\
\hline Willick $1990 \ldots \ldots \ldots \ldots \ldots \ldots \ldots$. & Clusters & $\mathbf{R}$ & Phot & 0.29 & 7 & 0.30 & 0.10 \\
\hline Peletier \& Willner 1991 ......... & Virgo & $\mathbf{H}$ & Phot & 0.29 & 10 & 0.46 & 0.10 \\
\hline Bothun et al. 1992 .............. & Antlia and Hydra & I & Kin & 0.29 & 10 & 0.25 & 0.08 \\
\hline
\end{tabular}

a Photometric passband used.

b Phot indicates that the photometry was used to derive inclinations, and kin indicates that the inclination was derived from the gas kinematics.

c Minimum apparent ellipticity.

d Slope $a$ of the Tully-Fisher relation, defined as $M=-a^{10} \log v_{c}+b$.

e Scatter in magnitude.

f Resulting maximum allowed ellipticity of the potential 
spirals measured by Grosbøl (1985). He lists the apparent axial ratios at a radius $R \approx 3 R_{D}$, also gives the measurement errors. This is important, because such errors result in a systematic increase in measured apparent ellipticity at low true apparent ellipticity. We calculated the expected distribution for circular and elongated disks, and tested these with the KolmogorovSmirnov method. The distribution of apparent axial ratios is well fitted by models with $\epsilon_{D}=0.10-0.13$ in the plane of the disk. This result agrees with a similar analysis by Huizinga \& van Albada (1992) of an independent sample of Sc galaxies. This photometric axial ratio corresponds to an ellipticity of the potential between $\epsilon=0.08$ and $\epsilon=0.10$. Grosbøl's sample is diameter limited, so that it is unbiased with respect to ellipticity if galaxies have large internal absorption. We defined a magnitude-limited subsample, which is unbiased if galaxies are transparent. The best-fitting model now has $\epsilon_{D}=0.06$.

The above analyses assume that the error distribution in apparent ellipticity is Gaussian. This may not be realistic for small ellipticities, where measurement errors are expected to systematically increase the observed ellipticity. We therefore use equation (2) of Franx, Illingworth, \& Heckman (1989) to derive a more realistic error distribution. In this case circular models $\left(\epsilon_{D}=0\right)$ give the best fit. We conclude that the photometry puts an upper limit on the ellipticity of the potential of $\epsilon=0.10$. Even if more detailed analyses confirm photometric ellipticities of 0.12 , then these should still be regarded as an upper limit to the systematic elongation of disks. It is well known that spiral arms can be quite strong in the outer parts (e.g., Wevers, van der Kruit, \& Allen 1986), and these are hard to distinguish from a systematic elongation without detailed modeling of the two-dimensional surface photometry.

The constrants from the photometry and from the scatter in the Tully-Fisher relation are in agreement, and indicate an upper limit on the ellipticity of the potential of $\epsilon=0.10$. However, there are strong reasons to suspect that this value is still too large. Most importantly, it is very unlikely that all the observed scatter in the Tully-Fisher relation is caused by this one effect. The internal properties of spiral galaxies show quite a bit of spread (e.g., Kennicutt 1983; Pierce \& Tully 1988), which must cause scatter in the internal relation between $L$ and $v_{c}$. Furthermore, the observed dispersion in the Tully-Fisher relation is increased by true distance differences between galaxies in the same cluster. As an example, Tonry, Ajhar, \& Luppino (1990) found a distance scatter for ellipticals in the direction of Virgo of $22 \%$, corresponding to a scatter of 0.43 mag. They argued that most of this scatter was due to galaxies projected onto the cluster. Hence, it is more reasonable to assume that maximally half the scatter in the Tully-Fisher relation is due to the ellipticity of the potential. This implies that the ellipticity $\epsilon$ lies between 0 and 0.06 .

\section{DISCUSSION}

We have shown that a moderate elongation of the gravitational potential in the disk plane leads to observable effects on the photometry and kinematics of spiral galaxies. The presence of bulges, bars, spiral arms, and dust confuse the photometric signatures, however.

The strongest constraint on the elongation of the potential comes from the small observed scatter in the Tully-Fisher relation. Assuming that all observed scatter is due to the elongation of the potential, we find ellipticities between 0.08 and 0.10 . The differences in observed scatter are naturally explained by the differences in the methods used in the various studies. These ellipticities are firm upper limits, as there must be other sources of scatter in the Tully-Fisher relation. If maximally half the scatter in the Tully-Fisher relation is due to elongated potentials, the ellipticity lies between 0 and 0.06 . Such an ellipticity contributes a scatter of $0.26 \mathrm{mag}$ in the Tully-Fisher relation if photometric inclinations are used, and $0.18 \mathrm{mag}$ if kinematic inclinations are used (Fig. 2).

The elongation of the potential can be measured by a careful analysis of kinematic maps of spiral galaxies. If the ellipticity is anywhere between 0.05 and 0.10 , the measurement of the ellipticity of individual disks may lead to a reduction in the scatter of the Tully-Fisher relation of $0.15-0.45 \mathrm{mag}$.

It is still difficult to constrain the shapes of dark halos with this result, as we do not know very well what the relative contributions of the dark halo and the luminous material are. If the halo dominates the potential, then an $\epsilon=0.06$ corresponds to a density distribution with an ellipticity in the disk plane equal to 0.16 . However, it is often argued that the disk and the halo contribute roughly equally to the potential (van Albada 1988). In this case the constraint is much weaker, as the disk will tend to be elongated in a direction opposite to the elongation of the halo. As a result, we obtain a weak upper limit on the ellipticity of the halo density in the plane of the disk of 0.41 .

We conclude that the potentials of spiral galaxies have small systematic elongations in the disk plane, with ellipticity most likely in the range $0-0.06$, but certainly below 0.10 .

It is a pleasure to thank Koen Kuijken, Joanna Lees, HansWalter Rix, Michael Rupen, and Martin Schwarzschild for stimulating conversations and comments on the manuscript. Preben Grosbøl kindly made his data available in digital form. M. F. was supported by a Hubble Fellowship. Travel support by het Leidsch Kerkhoven-Bosscha Fonds is gratefully acknowledged.

Aaronson, M., Bothun, G., Mould, J., Huchra, J., Schommer, R. A., \& Cornell,

M. E. 1986, ApJ, 302, 536

Begeman, K. 1987, Ph.D. thesis, Univ. of Groningen

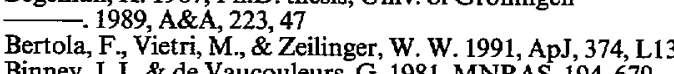

Binney, J. J., \& de Vaucouleurs, G. 1981, MNRAS, 194, 679

Binney, J. J., \& Tremaine, S. D. 1987, Galactic Dynamics (Princeton: Princeton Univ Press)

Bosma, A. 1981, AJ, 86, 1825

Bothun, G., \& Mould, J. R. 1987, ApJ, 313, 629

Bothun, G. D., Schommer, R. A., Williams, T. B., Mould, J. R., \& Huchra, J. P 1992, ApJ, 388, 253

Dubinski, J., \& Carlberg, R. G. 1991, ApJ, 378, 496

Franx, M., Illingworth, G. D., \& Heckman, T. M. 1989, AJ, 98, 538

Franx, M., van Gorkom, J. H., \& de Zeeuw, P. T. 1992, in preparation

Frenk, C. S., White, S. D. M., Davis, M., \& Efstathiou, G. 1988, ApJ, 327, 507

Gerhard, O. E., \& Vietri, M. 1986, MNRAS, 223, 377

\section{REFERENCES}

Grosbøl, P. J. 1985, A\&AS, 60, 261

Huizinga, J. E., \& van Albada, T. S., 1992, MNRAS, 254, 677

Kennicutt, R. C. 1983, ApJ, 272, 54

Kuijken, K., \& Tremaine, S. D. 1991, in Dynamics of Disk Galaxies, ed. B. Dundelius, (Göteborg: Göteborg Univ. Press), 71

Kraan-Korteweg, R. C., Cameron, L. M., \& Tammann, G. A., 1988, ApJ, 331, 620

Lees, J. F., \& de Zeeuw, P. T. 1992, in preparation

Peletier, R., \& Willner, S. 1992, ApJ, 382, 382

Pierce, M. J., \& Tully, R. B. 1988, ApJ, 330, 579

Tonry, J. L., Ajhar, E. A., \& Luppino, G. A., 1990, AJ, 100, 1416

Tully, R. B., \& Fischer, J. R. 1977, A\&A, 54, 661

van Albada, T. S. 1988, in Space Science and Fundamental Physics (ESA-SP 283), 165

Wevers, B. M. H. R., van der Kruit, P. C., \& Allen, R. J., 1986, A\&AS, 66, 505

White, S. D. M., \& Ostriker, J. P. 1990, ApJ, 349, 22

Willick, J. A. 1990, ApJ, 351, L5 